\title{
Expression of ING4 is negatively correlated with cellular proliferation and microvessel density in human glioma
}

\author{
BIN SHAO and ENZHONG LIU \\ Department of Neurosurgery, The First Affiliated Hospital of Harbin Medical University, \\ Harbin, Heilongjiang 150001, P.R. China
}

Received August 24, 2015; Accepted December 6, 2016

DOI: $10.3892 / \mathrm{ol} .2017 .6618$

\begin{abstract}
The present study aimed to investigate the associations of tumor inhibitor of growth 4 (ING4) with tumor cell proliferation and microvessel density (MVD) in human glioma. Semi-quantitative reverse transcription polymerase chain reaction, western blotting and immunohistochemistry (IHC) were used to detect the level of ING4 expression in normal brain tissues and glioma tissues of different pathological grades. The cell proliferation index (PI) and the level of MVD were detected using IHC, facilitating the analysis of the correlation between ING4 and cellular proliferation and MVD in human glioma. The expression levels of ING4 mRNA and protein were significantly lower in the glioma tissues compared with the normal brain tissues $(\mathrm{P}<0.05)$ and decreased as the pathological grade of the glioma tissue increased $(\mathrm{P}<0.05)$. PI was reduced in the ING4 protein positively expressed $\left(\mathrm{ING}_{4}{ }^{+}\right)$glioma tissues compared with the ING4 protein negatively expressed (ING4) glioma tissues $(\mathrm{P} \leq 0.01)$. The level of MVD was significantly higher in the glioma tissues than that in the normal brain tissues $(\mathrm{P} \leq 0.01)$, while the level of MVD decreased in the $\mathrm{ING}^{+}$glioma tissue compared with the ING4- glioma tissue $(\mathrm{P} \leq 0.001)$. A Spearman's rank correlation test revealed that ING4 protein expression was negatively correlated with PI and MVD. ING4 might inhibit tumor proliferation and angiogenesis in human glioma. The expression of ING4 was also associated with the pathological grading of the glioma tissue and negatively correlated with cellular proliferation and MVD in human glioma.
\end{abstract}

\section{Introduction}

High-grade glioma is the most common type of intracranial malignant tumor and is characterized by the rapid proliferation

Correspondence to: Dr Enzhong Liu, Department of Neurosurgery, The First Affiliated Hospital of Harbin Medical University, 199 East Dazhi Street, Nangang, Harbin, Heilongjiang 150001, P.R. China E-mail: enzhongliuezl@163.com

Key words: human glioma, tumor inhibitor of growth 4, proliferation index, microvessel density and invasion of the tumor (1). At present, there are certain conventional therapeutic methods available for patients with high-grade glioma, including neurosurgical resection, radiochemotherapy and polychemotherapy. However, the 5-year survival rate is less than $3 \%$ (2). Thus, the study of the molecular mechanisms of high-grade glioma and the development of effective methods of diagnosis and treatment is essential.

Inhibitor of growth (ING) 4 is a vital tumor suppressor factor. Previous studies found that ING4 performed an important role in inhibiting tumor growth, promoting tumor apoptosis, recovering intercellular contact inhibition, affecting cell cycle progression and inhibiting tumor angiogenesis (3-6). In vitro experiments revealed that ING4 was abundantly expressed in normal tissues, but expression was significantly reduced in various malignant tumors, including melanoma (7), ovarian cancer (8), lung cancer (9) and glioma (10).

Malignant tumor growth and angiogenesis are largely caused by the generation and rapid growth of new blood vessels, with angiogenesis reported as the hallmark of malignant tumors including high-grade glioma $(11,12)$. The microvessel density (MVD) of human tissues has been reported as an important indicator in the evaluation of angiogenesis (13). A previous study suggested that ING4 could suppress brain tumor growth and angiogenesis by regulating the nuclear factor $\kappa \mathrm{B}$ cells signaling pathway (10). In addition, Zhao et al (14) have demonstrated that ING4 expression has an important clinical significance in human brain astrocytoma. However, the association between ING4 and cellular proliferation and MVD has not been investigated.

In the present study, semi-quantitative reverse transcription polymerase chain reaction (RT-PCR) western blotting and immunohistochemistry (IHC) methods were performed to detect the expression levels of ING4 in normal brain tissue and glioma tissues of different pathological grades. The correlations between the level of ING4 expression and human glioma pathological grading, cellular proliferation and MVD were then analyzed, aiming to reveal the mechanism of how ING4 is associated with glioma.

\section{Materials and methods}

Patients. The present study recruited a total of 85 patients with glioma at The First Affiliated Hospital of Harbin Medical University (Harbin, China) between January 2012 
and September 2013. The patients, including 29 males and 56 females, were aged between 20 and 71 years and had a median age of 41 years, with complete clinicopathological records. The pathological grading of the glioma tissues was performed according to The World Health Organization (WHO) 2008 Classification of Tumours of the Central Nervous System (15). Among the 85 patients, 11 patients were classified as grade I, 18 as grade II, 41 as grade III and 15 as grade IV. None of the patients received chemotherapy or radiotherapy prior to surgery, and the glioma tissue was collected during surgery. Normal brain tissue adjacent to the brain tumors was used as the negative control. The present study was approved by the Ethics Committee of Harbin Medical University (Harbin, China). Written informed consent was obtained from all participants.

All specimens were freshly collected using RNase-free techniques. The specimens were divided into 2 sections: The first section was immediately stored in liquid nitrogen for subsequent use in the semi-quantitative RT-PCR and western blotting, and the other section was fixed in $10 \%$ formalin solution for the IHC.

RNA isolation, reverse transcription and semi-quantitative $R T-P C R$. The neuronal tissue was homogenized on ice with TRIzol reagent (Invitrogen; Thermo Fisher Scientific, Inc., Waltham, MA, USA), and the RNA was extracted according to the manufacturers protocol. High-quality RNA was reverse transcribed into complementary DNA (cDNA) using a reverse transcription kit (Promega Corporation, Madison, WI, USA). The primers were: ING4 forward, 5'-CACAAGTCCTGAGTA TGGGAT-3' and reverse, 5'-AGGGGATGTGGAAGAAAC TGT-3'; GAPDH forward, 5'-CAGGGCTGCTTTTAACTC TG-3' and reverse, 5'-CTGTTGTCGGAGTTCTAGTAG-3'. The PCR reaction mixes were composed of $10 \mathrm{ng}$ cDNA, $0.1 \mu \mathrm{M}$ primers, $1 \mathrm{mM}$ deoxy-ribonucleoside triphosphate, 5 U Taq DNA Polymerase (Promega Corporation), $2.5 \mu 1$ 10X buffer, and double distilled water for a final volume of $25 \mu \mathrm{l}$. A reaction system without cDNA served as a negative control. The PCR program was as follows: $95^{\circ} \mathrm{C}$ for $3 \mathrm{~min}, 28$ cycles of $95^{\circ} \mathrm{C}$ for $10 \mathrm{sec}, 55^{\circ} \mathrm{C}$ for $30 \mathrm{sec}$ and $72^{\circ} \mathrm{C}$ for $30 \mathrm{sec}$. The PCR products were separated by $2 \%$ agarose gel electrophoresis. Ethidium bromide at a concentration of $0.5 \mathrm{mg} / \mathrm{ml}$ (Sigma-Aldrich; Merck Millipore, Darmstadt, Germany) was used to stain the DNA for visualization using a UV transilluminator. The PCR products were analyzed using gel analysis software Quantity One version 4.4 (Bio-Rad Laboratories, Inc., Hercules, CA, USA), and the data were used to measure corresponding optical density ratios (ING4 optical density value/GAPDH optical density value). The experiment was repeated 3 times to calculate the mean value.

Western blotting. The neural tissues were milled and then placed in radioimmunoprecipitation assay lysis buffer (Beyotime Institute of Biotechnology, Haimen, China) on ice for $30 \mathrm{~min}$. The supernatant was acquired by centrifugation at $10,000 \times \mathrm{g}$ for $15 \mathrm{~min}$ at $4^{\circ} \mathrm{C}$ to facilitate protein quantitation. The proteins were separated on 10\% SDS-PAGE gel and transferred onto polyvinylidene fluoride membranes. The membranes were blocked in 5\% non-fat milk diluted with PBS for $1 \mathrm{~h}$. Subsequently, the membranes were incubated overnight at $4^{\circ} \mathrm{C}$ with rabbit anti-human ING4 polyclonal antibody (dilution, 1:200; \#ab113425, Abcam, Cambridge, UK) or mouse anti-human $\beta$-actin monoclonal antibody (dilution, 1:200; \#ab6276, Abcam), washed 3 times with PBS, and incubated with goat anti-mouse or mouse anti-rabbit IgG $(\mathrm{H}+\mathrm{L})$-horseradish peroxidase (HRP; dilution, 1:200; \#ab97240 and \#ab99702, Abcam, respectively) for $2 \mathrm{~h}$ at room temperature. The proteins were detected using enhanced chemiluminescence (EMD Millipore, Billerica, MA, USA) and protein expression was quantified using Quantity One version 4.4 software (Bio-Rad Laboratories, Inc.). The experiment was repeated 3 times.

Detection of ING4 and Ki-67 expression by IHC. The specimens were fixed in $10 \%$ formalin solution for $24 \mathrm{~h}$, embedded into paraffin blocks, and then sliced into $4 \mu \mathrm{m}$ thick sections. The sections were deparaffinized for $4 \mathrm{~h}$ at $65^{\circ} \mathrm{C}$ and dehydrated with gradient ethanol. IHC was performed using the two-step immunohistochemistry detection kit according to the manufacturers protocol (Harbin Hongbo Biotech Co., Ltd, Harbin, China). In brief, the sections were incubated with $3 \%$ $\mathrm{H}_{2} \mathrm{O}_{2}$ to block the endogenous peroxidase activity for $10 \mathrm{~min}$ at room temperature. An antigen retrieval was performed in citrate buffer $(10 \mathrm{mM}, \mathrm{pH} 6.0)$ for $10 \mathrm{~min}$ at $95^{\circ} \mathrm{C}$. The sections were then incubated with rabbit anti-human ING4 polyclonal antibody (dilution, 1:200; \#ab113425, Abcam), mouse anti-human Ki-67 monoclonal antibody (dilution, 1:200; \#ab8191, Abcam) and mouse anti-human tumor necrosis factor receptor superfamily member 8 (CD34) monoclonal antibody (dilution, 1:200; \#ab8536, Abcam) overnight. The sections were washed with PBS, and subsequently incubated with goat anti-mouse or mouse anti-rabbit $\operatorname{IgG}(\mathrm{H}+\mathrm{L})-\mathrm{HRP}$ (dilution, 1:200; \#ab97240 and \#ab99702, Abcam, respectively) at $37^{\circ} \mathrm{C}$ for $30 \mathrm{~min}$. Finally, the sections were stained with diaminobenzidine (Harbin Hongbo Biotech Co., Ltd, Harbin, China) for $5 \mathrm{~min}$ and re-stained with hematoxylin for $2 \mathrm{~min}$. Sections of predetermined positive glioma tissue were used as the positive control, and PBS was used to replace the primary antibody in the negative controls.

Cells were considered to be ING4 positive when the nucleus stained brown-yellowish. A total of 4 fields were randomly selected and observed at a magnification of $\mathrm{x} 400$, and 100 cells were counted in each field. The expression of ING4 was assessed by a subjectively graded scale according to a previous study (16): Absence of positive cells were recorded as negative (-); $\leq 25 \%$ positive cells were recorded as weakly positive (+); $25-50 \%$ positive cells were recorded as moderately positive (++); and positive cells $>50 \%$ were recorded as strongly positive (+++).

The positive standard of the Ki-67 stain was set as the appearance of brown particles inside the nuclei of the tumor cells, 4 representative visions were selected at a magnification of $\mathrm{x} 400$. The proliferation index (PI) of the Ki-67 strain was calculated as: PI $(\%)=$ number of positive cells/cell number $\mathrm{x} 100$.

CD34 is a marker of vascular endothelial cells and was used to indicate the presence blood vessels. Positive CD34 expression located in the cytoplasm or membrane of vascular endothelial cells was used for quantifying MVD. Blood vessel distribution was observed under a light microscope (BX41, 
Olympus Corporation, Tokyo, Japan) at a magnification of $\mathrm{x} 100$, and the area with the highest density of microvessels was selected to count microvascular numbers at a magnification of $\mathrm{x} 200$ in 4 sections. The mean of the 4 sections was used as the MVD for this tissue section.

Statistical analyses. Statistical analyses were performed using SPSS 18.0 statistics software (SPSS Inc., Chicago, IL, USA). Statistical analyses of the mRNA and protein expression levels of ING4, MVD and PI in normal tissues and various grades of glioma tissues were performed by a Wilcoxon rank sum test. Data are expressed as the mean \pm the standard deviation. The ING4 positive expression rate in normal tissue and tissue with various grades of glioma was analyzed using a $\chi^{2}$ test. The correlation of ING4 expression levels with PI and MVD was analyzed by Spearman's rank correlation. $\mathrm{P}<0.05$ was considered to indicate a statistically significant difference.

\section{Results}

Semi-quantitative RT-PCR and western blotting analysis revealed that the mRNA $(\mathrm{P}=0.015)$ and protein $(\mathrm{P}=0.0123)$ expression levels of ING4 in glioma brain tissue were lower than that of the normal brain tissue (Table I). IHC with anti-ING4 antibody showed that the expression of ING4 protein in the nucleus presented as brown-yellowish granules and indicated that the ING4 positive expression rate significantly decreased in the glioma tissues compared with the normal brain tissue $(\mathrm{P}=0.01$; Table I). The mRNA and protein expression levels of ING4 were compared based on age, gender, tumor size and pathological grading. The results revealed that the expression of ING4 was not correlated with age, gender or tumor size in the glioma tissues (Table I). However, there was a significant decrease in ING4 mRNA ( $\mathrm{P}=0.026$, Fig. 1A) and protein $(\mathrm{P}=0.003$, Fig. $1 \mathrm{~B})$ expression as the pathological grade increased (Table I). The ING4 positive expression rate also decreased as the pathological grade increased $(\mathrm{P}=0.015$, Fig. 1C; Table I).

IHC with anti-CD34 antibody showed that CD34-labeled microvessels existed in the normal brain and glioma tissues, with the level of MVD higher in the glioma brain tissues compared with the normal brain tissues $(\mathrm{P}=0.011$, Table I). The level of MVD was not correlated with age, gender or tumor size in the glioma tissues, however, MVD level significantly increased as the pathological grades increased ( $\mathrm{P}=0.0018$; Fig. 2; Table I). The level of MVD was lower in the ING4 protein positively expressed $\left(\mathrm{ING}^{+}\right)$glioma tissues $(17.35 \pm 4.12)$ compared with the ING4 protein negatively expressed (ING4 ${ }^{-}$) glioma tissues (22.95 \pm 1.88 ; $\mathrm{P} \leq 0.001$; Table II).

In addition, IHC with anti-Ki-67 antibody revealed that $\mathrm{Ki}-67$ was expressed in the nucleus. PI decreased in the ING4+ glioma tissues $(25.98 \pm 4.67)$ compared with the ING4- glioma tissues (48.46 \pm 7.26$)(\mathrm{P}=0.01$ Fig. 3, Table II). Furthermore, the Spearman's rank correlation test demonstrated that ING4 protein expression was negatively correlated with PI ( $\mathrm{r}=-0.614$, $\mathrm{P}=0.035$ ). The positive expression rate of the ING4 gene in different pathologically graded glioma tissues was negatively correlated with MVD ( $\mathrm{r}=-0.825, \mathrm{P}=0.004)$ while PI and MVD in the glioma tissue were positively correlated $(r=0.364$, $\mathrm{P}=0.023)$.

\section{Discussion}

High-grade glioma is the most common of the primary brain tumors in adults, with a high incidence rate and poor prognosis (2). The present study demonstrated that the expression level of ING4 was lower in glioma brain tissue compared with normal brain tissue. Furthermore, there was a significant decrease in the levels of ING4 mRNA and protein expression as the pathological grade of the glioma increased, suggesting that ING4 may perform an important role in the occurrence and progression of the tumor. In addition, PI and MVD were lower in the ING4+ $4^{+}$glioma tissues compared with the ING4- glioma tissues, which demonstrated that ING4 protein expression was negatively correlated with PI and MVD in human glioma.

Previous studies revealed that the downregulation of ING4 was involved in the progression of hepatocellular carcinoma tissue (17), head and neck squamous cell carcinoma (18), human astrocytomas (19) and colorectal cancer (20). Consistent with the results of the present study, Gong et al (21) suggested that the expression of ING4 in glioma tissue was inhibited, resulting in the inhibition of tumor cell growth and apoptosis by autophagy. In addition, Colla et al (22) reported that ING4 exhibits 4 spliceosomes, of which 3 spliceosomes had no complete nuclear localization signals and were more frequently located in the nucleus. The results of the IHC of the present study also demonstrated that the ING4 protein was mainly expressed inside the nucleus. Additionally, the present study found that the ING4 positive rate decreased as the pathological grading increased, but was independent from gender, age and tumor size, indicating that ING4 protein expression in glioma tissue was associated with the degree of malignancy of the glioma, which are similar to the results of a study examining the role of ING4 in colon cancer (23).

Furthermore, as a result of the present study it was hypothesized that ING4 inhibits glioma proliferation. Zhang et al (24) found that ING4 could negatively regulate cell growth by arresting the cell cycle during the G2/M phase and increasing the sensitivity of HepG2 cells to certain DNA-damaging agents. In addition, a different study indicated that the overexpression of ING4 may negatively regulate U251 cell proliferation by enhancing the binding of the cells to p53 and increasing levels of p21 expression to induce cell cycle arrest (25). In addition, angiogenesis was a feature of a number of tumors and involved with the growth, invasion, and metastasis of cancer cells (12). The level of MVD has been used as the gold standard in terms of evaluating the state of angiogenesis of solid tumors (26). The number of vessels was significantly higher in human glioma compared with normal brain tissue (27). The present study suggested that the suppression of ING4 expression in glioma tissue was associated with the formation of new blood vessels. A previous study also showed that the level of MVD was significantly higher in glioma cells transfected with antisense ING4 compared with control cells (10). In addition, ING4 inhibited angiogenesis via interleukin-8 in human glioblastoma (28). The present study also revealed that the expression level of ING4 was negatively correlated with PI and MVD. Additionally, it was demonstrated that PI was positively correlated with the level of MVD in glioma tissue, indicating that when the expression of ING4 was suppressed in the glioma tissue, tumor cell proliferation and the formation 


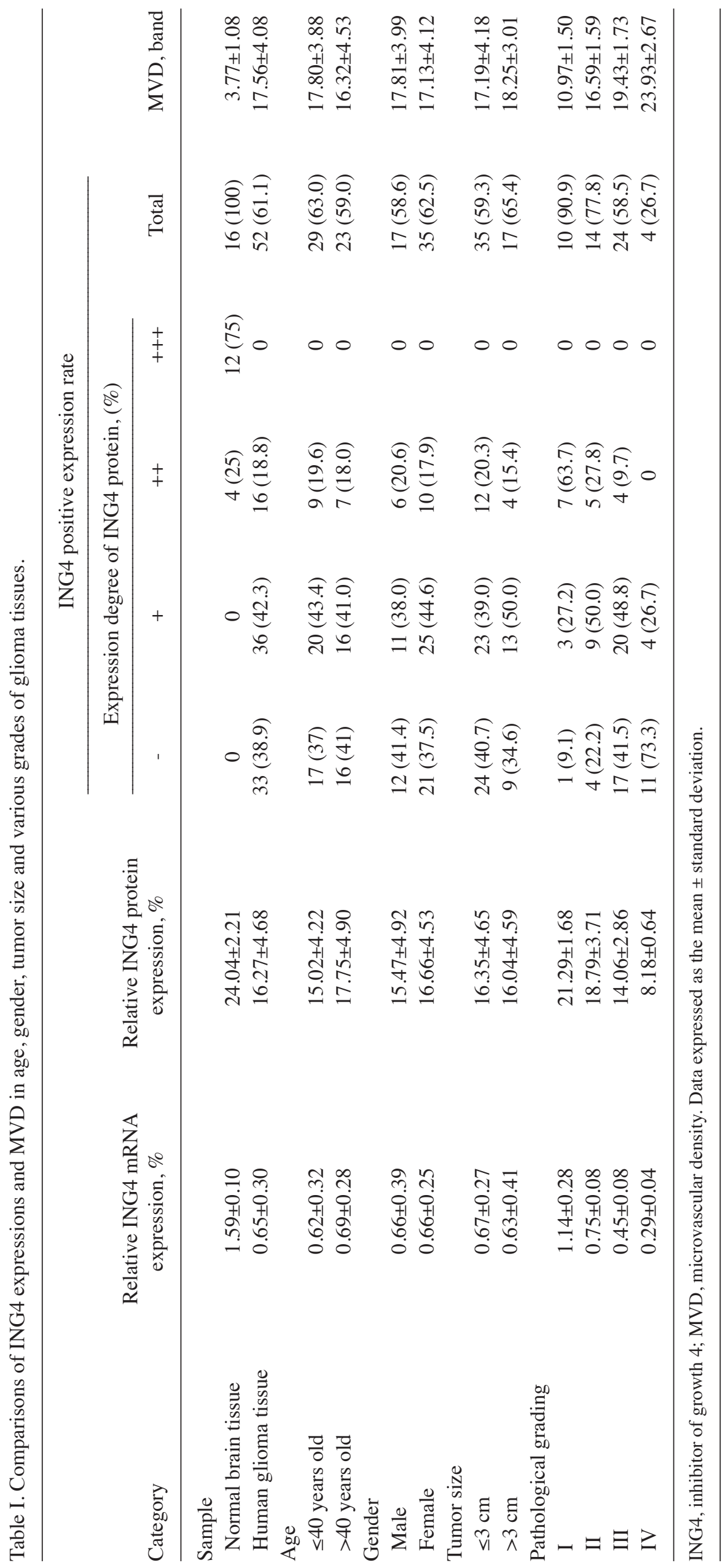


Table II. MVD and PI in ING4 positively and negatively expressed glioma tissues.

\begin{tabular}{lcrr}
\hline & ING4 negatively expressed tissues $(\mathrm{n}=33)$ & ING4 positively expressed tissues (n=52) & $\mathrm{t}$ \\
\hline MVD, band & $22.95 \pm 1.88$ & $17.35 \pm 4.12$ & 14.623 \\
PI, \% & $48.46 \pm 7.26$ & $25.98 \pm 4.67$ & 0.001 \\
\hline
\end{tabular}

ING4, inhibitor of growth 4; MVD, microvascular density; PI, proliferation index. Data expressed as the mean \pm standard deviation.

A

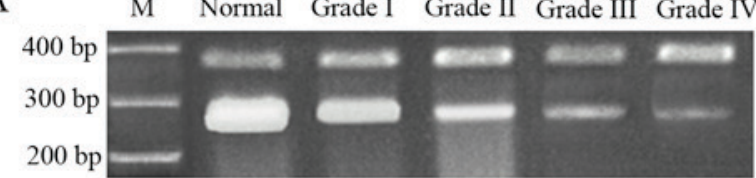

B Normal Grade I Grade II Grade III Grade IV

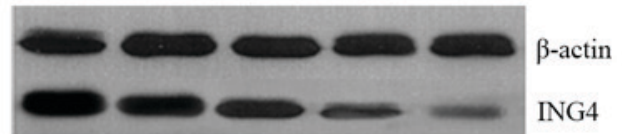

C Grade I GAPDH
ING4 Grade II Grade III Grade IV

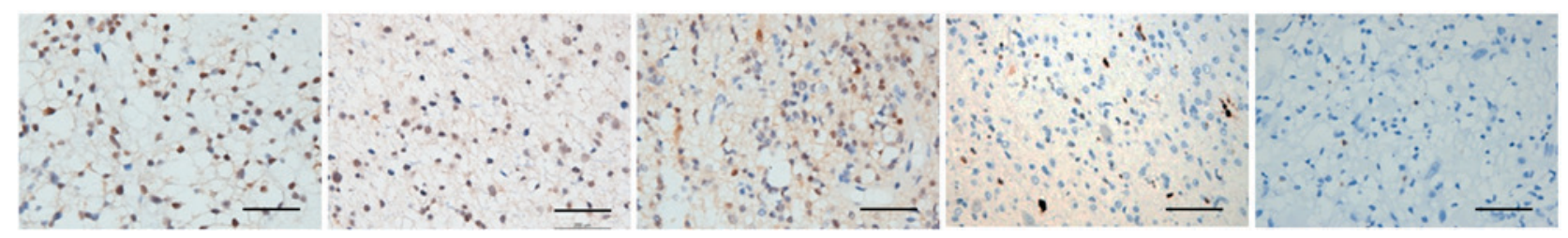

Figure 1. Expression of ING4 in different glioma and normal brain tissues. (A) Semi-quantitative PCR, (B) western blotting and (C) immunohistochemistry analyses revealed that the expression of ING4 was lower in the glioma brain tissue than in the normal brain tissue and decreased with increasing pathological grades. Scale bar, $100 \mu \mathrm{M}$. ING4, inhibitor of growth 4; M, DNA marker; Normal, normal brain tissue; Grade I, glioma of pathological grade I; Grade II, glioma of pathological grade II; Grade III, glioma of pathological grade III; Grade IV, glioma of pathological grade IV.

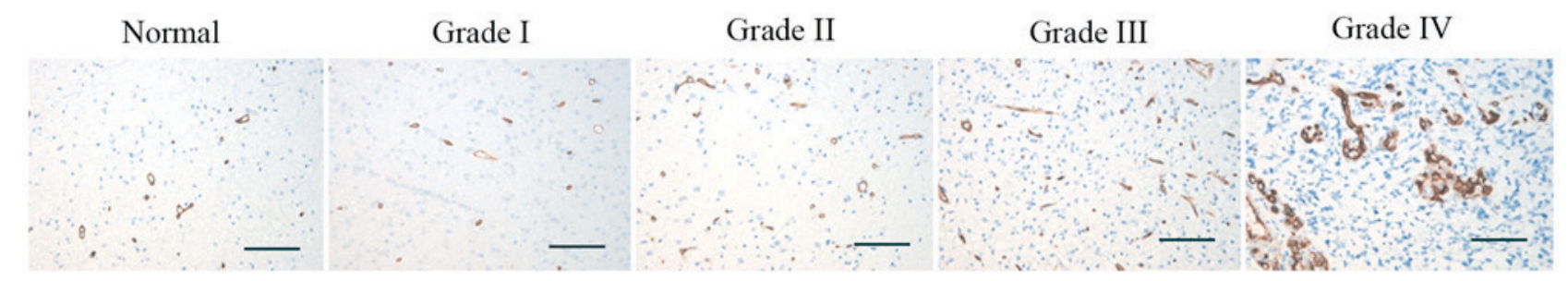

Figure 2. Expression of CD34 in different glioma and normal brain tissues. The number of CD34-labeled microvessels increased in the glioma brain tissue compared with the normal brain tissue, and increased with increasing pathological grades. Scale bar, 100 $\mu \mathrm{M}$. CD34, hematopoietic progenitor cell antigen.

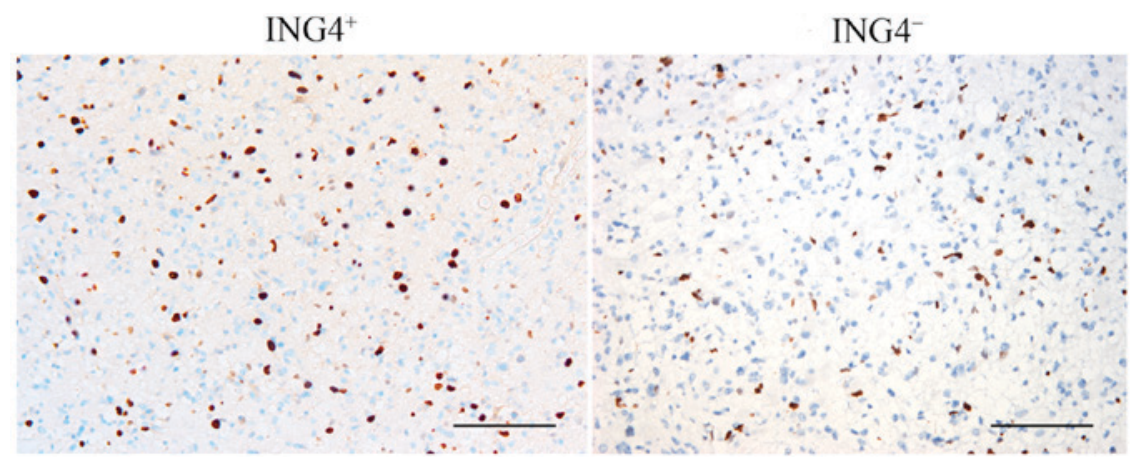

Figure 3. Expression of Ki-67 in different glioma and normal brain tissues. The number of Ki-67 positively expressed cells decreased in the ING4 protein positively expressed glioma tissues compared with the ING4 protein negatively expressed glioma tissues. ING4, inhibitor of growth 4; ING4; ING4 protein negatively expressed glioma tissues; ING4 ${ }^{+}$, ING4 protein positively expressed glioma tissues. Scale bar, $10 \mu \mathrm{m}$.

of tumor microvessels was promoted and ING4 was negatively associated with the degree of malignancy of the glioma. Similar to the results of the present study, Lou et al (23) demonstrated that there was a negative correlation between the level of MVD and the level of ING4 expression in patients with colorectal carcinoma, and a higher tumor node metastasis 
stage was correlated with a lower level of ING4 expression and a higher level of MVD. A limitation of the present study was that the mechanism underlying ING4-mediated regulation of cell proliferation and angiogenesis in high-grade glioma is not fully understood. Future studies are required to investigate this mechanism.

In conclusion, the occurrence and development of glioma is a multi-stage process and involves multiple gene changes. ING4 inhibited the proliferation and angiogenesis of glioma. The expression of ING4 was associated with the pathological grading of the glioma tissue and negatively correlated with cell proliferation and angiogenesis, while tumor proliferation and angiogenesis were positively correlated in the glioma tissues.

\section{Acknowledgements}

The present study was supported by Heilongjiang Provincial Health Department Research Projects (grant no. 2007-534).

\section{References}

1. Wen PY and Kesari S: Malignant gliomas in adults. N Engl J Med 359: 492-507, 2008.

2. Ohgaki $\mathrm{H}$ and Kleihues P: Epidemiology and etiology of gliomas. Acta Neuropathol 109: 93-108, 2005.

3. Aggarwal R, Lu J, Kanji S, Das M, Joseph M, Lustberg MB Ray A, Pompili VJ, Shapiro CL and Das H: Human V $\gamma 2$ V $\Delta 2 \mathrm{~T}$ cells limit breast cancer growth by modulating cell survival-, apoptosis-related molecules and microenvironment in tumors. Int J Cancer 133: 2133-2144, 2013.

4. Hou Y, Zhang Z, Xu Q, Wang H, Xu Y and Chen K: Inhibitor of growth 4 induces $\mathrm{NF} \kappa \mathrm{B} / \mathrm{p} 65$ ubiquitin-dependent degradation. Oncogene 33: 1997-2003, 2014.

5. Culurgioni S, Muñoz IG, Moreno A, Palacios A, Villate M, Palmero I, Montoya G and Blanco FJ: Crystal structure of inhibitor of growth 4 (ING4) dimerization domain reveals functional organization of ING family of chromatin-binding proteins. J Biol Chem 287: 10876-10884, 2012.

6. Zhao Y, Li Z, Sheng W, Miao J and Yang J: Adenovirus-mediated ING4/IL-24 double tumor suppressor gene co-transfer enhances antitumor activity in human breast cancer cells. Oncol Rep 28 : 1315-1324, 2012

7. Tang Y, Cheng Y, Martinka M, Ong CJ and Li G: Prognostic significance of KAI1/CD82 in human melanoma and its role in cell migration and invasion through the regulation of ING4 Carcinogenesis 35: 86-95, 2014.

8. Liu Y, Yu L, Wang Y, Zhang Y, Wang Y and Zhang G: Expression of tumor suppressor gene ING4 in ovarian carcinoma is correlated with microvessel density. J Cancer Res Clin Oncol 138: 647-655, 2012

9. Ling C, Xie Y, Zhao D, Zhu Y, Xiang J and Yang J: Enhanced radiosensitivity of non-small-cell lung cancer (NSCLC) by adenovirus-mediated ING4 gene therapy. Cancer Gene Ther 19: 697-706, 2012

10. Garkavtsev I, Kozin SV, Chernova O, Xu L, Winkler F, Brown E, Barnett GH and Jain RK: The candidate tumour suppressor protein ING4 regulates brain tumour growth and angiogenesis. Nature 428: 328-332, 2004.

11. Bhattacharya A, Turowski SG, San Martin ID, Rajput A, Rustum YM, Hoffman RM and Seshadri M: Magnetic resonance and fluorescence-protein imaging of the anti-angiogenic and anti-tumor efficacy of selenium in an orthotopic model of human colon cancer. Anticancer Res 31: 387-393, 2011.
12. Folkman J: How is blood vessel growth regulated in normal and neoplastic tissue? G.H.A. clowes memorial award lecture. Cancer Res 46: 467-473, 1986.

13. Rubatt JM, Darcy KM, Hutson A, Bean SM, Havrilesky LJ, Grace LA, Berchuck A and Secord AA: Independent prognostic relevance of microvessel density in advanced epithelial ovarian cancer and associations between CD31, CD105, p53status, and angiogenic marker expression: A Gynecologic Oncology Group study. Gynecol Oncol 112: 469-474, 2009.

14. Zhao S, Jin C, Zhao X, Jin B, Hui L, Zhou W, Niu G and Tao S: Expression and clinical significance of ING4 and HIF-1 alpha in brain astrocytoma. Zhonghua Yi Xue Za Zhi 95: 3533-3536, 2015 (In Chinese).

15. Fuller GN: The WHO classification of tumours of the central nervous system, 4th edition. Arch Pathol Lab Med 132: 906, 2008.

16. Liu H, Wan D, Pan Z, Cao L, Wu X, Lu Z and Kang T: Expression and biological significance of leptin, leptin receptor, VEGF, and CD34 in colorectal carcinoma. Cell Biochem Biophys 60: 241-244, 2011.

17. Fang F, Luo LB, Tao YM, Wu F and Yang LY: Decreased expression of inhibitor of growth 4 correlated with poor prognosis of hepatocellular carcinoma. Cancer Epidemiol Biomarkers Prev 18: 409-416, 2009.

18. Li XH, Kikuchi K, Zheng Y, Noguchi A, Takahashi H, Nishida T, Masuda S, Yang XH and Takano Y: Downregulation and translocation of nuclear ING4 is correlated with tumorigenesis and progression of head and neck squamous cell carcinoma. Oral Oncol 47: 217-223, 2011.

19. Klironomos G, Bravou V, Papachristou DJ, Gatzounis G, Varakis J, Parassi E, Repanti M and Papadaki H: Loss of inhibitor of growth (ING-4) is implicated in the pathogenesis and progression of human astrocytomas. Brain Pathol 20: 490-497, 2010.

20. You Q, Wang XS, Fu SB and Jin XM: Downregulated expression of inhibitor of growth 4 (ING4) in advanced colorectal cancers: A non-randomized experimental study. Pathol Oncol Res 17: 473-477, 2011.

21. Gong A, Ye S, Xiong E, Guo W, Zhang Y, Peng W, Shao G, Jin J, Zhang Z, Yang J and Gao J: Autophagy contributes to ING4-induced glioma cell death. Exp Cell Res 319: 1714-1723, 2013.

22. Colla S, Tagliaferri S, Morandi F, Lunghi P, Donofrio G, Martorana D, Mancini C, Lazzaretti M, Mazzera L, Ravanetti L, et al: The new tumor-suppressor gene inhibitor of growth family member 4 (ING4) regulates the production of proangiogenic molecules by myeloma cells and suppresses hypoxia-inducible factor-1 alpha (HIF-1alpha) activity: Involvement in myeloma-induced angiogenesis. Blood 110: 4464-4475, 2007.

23. Lou C, Jiang S, Guo X and Dong XS: ING4 is negatively correlated with microvessel density in colon cancer. Tumor Biol 33: 2357-2364, 2012

24. Zhang $\mathrm{X}, \mathrm{Xu}$ LS, Wang ZQ, Wang KS, Li N, Cheng ZH, Huang SZ, Wei DZ and Han ZG: ING4 induces G2/M cell cycle arrest and enhances the chemosensitivity to DNA-damage agents in HepG2 cells. FEBS Lett 570: 7-12, 2004.

25. Liu E, Wu J, Cao W, Zhang J, Liu W, Jiang X and Zhang X: Curcumin induces G2/M cell cycle arrest in a p53-dependent manner and upregulates ING4 expression in human glioma. J Neurooncol 85: 263-270, 2007.

26. Goodheart MJ, Ritchie JM, Rose SL, Fruehauf JP, De Young BR and Buller RE: The relationship of molecular markers of p53 function and angiogenesis to prognosis of stage I epithelial ovarian cancer. Clin Cancer Res 11: 3733-3742, 2005.

27. Luo Y, Jiang F, Cole TB, Hradil VP, Reuter D, Chakravartty A, Albert DH, Davidsen SK, Cox BF, McKeegan EM and Fox GB: A novel multi-targeted tyrosine kinase inhibitor, linifanib (ABT-869), produces functional and structural changes in tumor vasculature in an orthotopic rat glioma model. Cancer Chemother Pharmacol 69: 911-921, 2012.

28. Brat DJ, Bellail AC and Van Meir EG: The role of interleukin- 8 and its receptors in gliomagenesis and tumoral angiogenesis. Neuro Oncol 7: 122-133, 2005. 